\title{
Exploration and Analysis on the Feasibility of VR Panoramic Technology Applied to Practical Training Teaching in Colleges and Universities
}

\author{
Yinglong Yu, Guili Zhang, Hanyue Zhang \\ Southwest Petroleum University, Chengdu, China \\ Email: 33138395@qq.com
}

How to cite this paper: Yu, Y. L., Zhang, G. L., \& Zhang, H. Y. (2021). Exploration and Analysis on the Feasibility of VR Panoramic Technology Applied to Practical Training Teaching in Colleges and Universities. Advances in Applied Sociology, 11, 350-356.

https://doi.org/10.4236/aasoci.2021.118032

Received: July 7, 2021

Accepted: August 1, 2021

Published: August 4, 2021

Copyright $\odot 2021$ by author(s) and Scientific Research Publishing Inc. This work is licensed under the Creative Commons Attribution-NonCommercial International License (CC BY-NC 4.0).

http://creativecommons.org/licenses/by-nc/4.0/

\begin{abstract}
VR panoramic technology, which has become increasingly perfect, has gained an expansive area in its application fields. Based on detailed elaboration on the dilemma existing in the practical training teaching in colleges and universities, the article expounds the opportunities brought by VR panoramic technology to teaching, and the theoretical hardware and software support of VR panoramic technology applied to teaching. Then, further conclusion will made on the feasibility of VR panoramic technology applied to practical training teaching in colleges and universities, which targets further promotion for the effect of practical training teaching.
\end{abstract}

\section{Keywords}

VR, Panoramic Technology, Practical Training Teaching

\section{Overview of VR Panoramic Technology Application}

Generally speaking, VR virtual reality environment generally exists in two forms: the virtual scene in virtue of 3D scene and model; the virtual scene based on the construction of image and video. Employing 720 filming equipment for image and video acquisition, VR panoramic technology is technologically classified into the former range and special software, which is made for assembling, editing and publishing. These settings can eventually achieve a panoramic immersive interactive experience through VR observation equipment (Ma, Guo, Li, \& Zou, 2021). Functioning as an important branch of VR, VR panoramic technology has been rapidly and frequently applied in a number of technological areas, including film and television, real estate, sightseeing and tourism, live broadcast, commercial display, etc. (Zhao, 2017). The area of higher education is undoub- 
tedly included in its commitment.

\section{The Dilemma Encountered by the Practical Training Teaching in Colleges and Universities}

As times are developing and progressing, employers are demanding more students to take up their supersized hands-on practical abilities. Amidst this backdrop, the status and weight of practical training teaching in colleges and universities are enjoying a daily soaring. Despite that, it is still encountered with some challenges.

\subsection{The Limitations of Time and Space}

Take Southwest Petroleum University as an example (hereinafter referred to as our university), some of our majors are set with practical training courses, most of which are located in the remote areas. As is known, long-distance driving calls for time-consumption and labor-intension. In face of this reality, teachers choose to lead students to carry out practical training courses with a certain degree of danger. For example, you may encounter mudslides, personnel separated, thunderstorms and other unexpected situations. From a large scale, these natural hazards double the difficult factors of practical training teaching (Zhou, Yang, Zhang, \& Gong, 2021).

\subsection{Student Participation Is Not Deep and Lack of Practice}

Relatively speaking, the time schedule of the practical training course is relatively compact. As the teacher needs more time for the narration, the students, as a result, have less opportunity to explore and practice in depth, which makes it insufficient to explore and analyze the depth of participation of the whole course. Considering that the process is not reversible, they do not learn on the spot if students are deserted at some places; never can they return to the training base for the relearning.

\subsection{Poor Teaching Effects and Evaluations}

Practically speaking, the various restrictions of the practical training environment, which will make the teaching effect discounted, can also exert some effects upon the teaching evaluation to a certain extent. For example, after the practical training, you need to write out an experimental report to give a summary of the knowledge, skills and methods acquired from the practical training. In this process, there may be experimental reports plagiarized from each other, which will negatively reduce the teaching effects. In this respect, traditional practical training teaching assessment and evaluation is still at a relatively single stage, and the assessment of students' real practical ability is not reached a well-appropriate stage.

\subsection{Insufficient Personalization of Teaching}

For the personalization of teaching, it is a tough task for traditional "one-to-many" 
practical training teaching mode to achieve personalized teaching. Practical training teachers exhibit their values in virtue of rich teaching experience and knowledge reserve. More than that, they are also capable of controlling practical activities. Nonetheless, it is nearly impossible for them to take care of and tutor every student, letting alone implementing personalized teaching.

\section{Opportunities Brought by VR Panoramic Technology for Practical Training Teaching in Colleges and Universities}

\subsection{Break the Limitation of Time and Space by Cutting down Teaching Cost}

Including most of the sensory information in the scene, VR panoramic technology contributes a highly-restored scene of practical training teaching. That presents students an immersive feeling of being in the scene by breaking the time and space limitation of practical training as it is a good money saver which can save a lot of teaching costs. It is a big helper allowing students to repeat their learning process and meanwhile avoid all kinds of risks, etc.

\subsection{Strengthen Classroom Interaction and Enhance Students' Interest}

Nowadays, a large majority of interaction forms of classroom education are located at two-dimensional level, and the main teaching carriers are composed of video and audio text resources. VR panoramic technology is a good interpreter to $3 \mathrm{D}$ real-world or virtual perspective and an excellent simulator of real interactive scenes. These functions have eventually increased students' interest and fun in participation, as a traveler traversing among reality and virtual anytime and anywhere.

\subsection{Increase Multiple Assessments and Consolidate the Internalization of Knowledge}

It is a well-functional strategy to add exploration, analysis and practice to the VR panoramic system. Based on breaking the single situation of relying only on experimental reports for assessment, it is also an examiner of students' mastery of knowledge and skills more comprehensively Beyond that, it can focus on the internalization of knowledge and the mastery and application of skills, greatly enriching the evaluation dimension.

\subsection{Personalized Learning Pace}

It has been exemplified that VR panoramic technology has managed to boost "personalized learning" to a new stage. A typical instance is that when students enter a VR training scenario, the system places them in the actual environment through the next assessment on their individual skills on the basis of a series of interactions. At some key steps, the system is working as a precise instructor who helps them meet their learning requirements. Within this context, students are allowed to set their own learning pace, adjust the difficulty of the system, 
adapt to their learning progress, etc.

\section{Feasibility of VR Panoramic Technology in Practical Training Teaching in Colleges and Universities}

\subsection{Theoretical Basis of VR Panoramic Technology Applied to Teaching}

Academically speaking, the theoretical basis of VR panoramic technology in the application of the teaching of practical training in colleges and universities mainly refers to the contextual teaching method (Sun, 2007). Specifically, contextual teaching method means the teaching method with which teachers can introduce or create vivid and concrete, scenes in virtue of certain emotional color, image as the main body. Oriented to tutor the students into corresponding experience, the commitment aims to help them understand the relevant knowledge points by accelerating the mental function to grow and expand. Students' emotions changes in the learning process have both positive and negative effects on cognitive activities by motivating, reinforcing, and regulating students' cognitive activity. Positive emotional states can be a good driver for learning, while negative emotions can hinder and inhibit learning on the other hand. The VR panoramic technology can compose a novel and rich panoramic situation for students, and meanwhile mobilizing students' positive emotions to some degree. All these effects can stimulate their interest in learning, making them generate positive emotions that promote learning cognition which is able to improve the internalization of knowledge based on enhancing the teaching effect.

\subsection{Advantages of VR Panoramic Technology in Creating Situations}

The advantages in the application of VR (Figure 1) panoramic technology to create panoramic situations are mainly composed of the following three aspects (Ge, 2018).

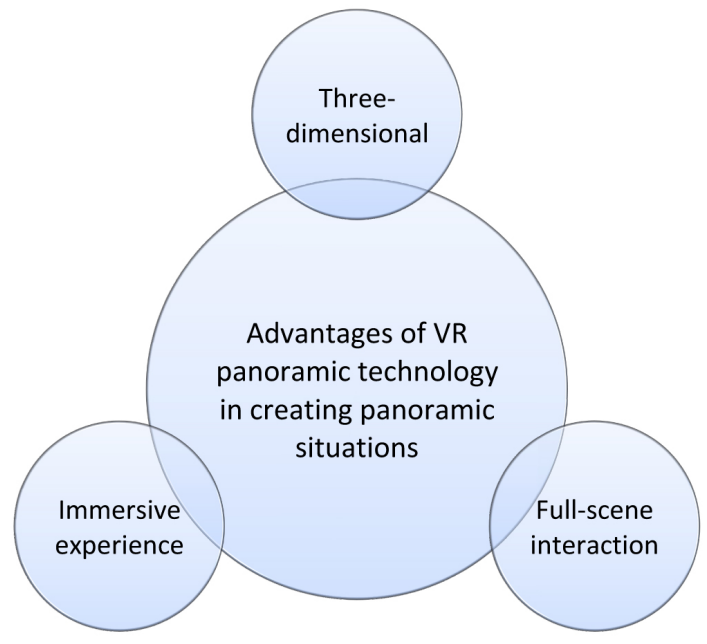

Figure 1. Advantages of VR panoramic technology in creating panoramic situations. 
1) Three-dimensional observation dimension. The panoramic situation composed by VR panoramic technology habours close connection with the observation dimension in the real world. The reason is that compared with the conventional pictures and videos which are limited to the two-dimensional observation level, VR panoramic is an impressive addition to a vertical level by structuring and shaping a three-dimensional observation dimension which widens the observation perspective and enables the situation to be more realistic.

2) Immersive experience. This mainly embraces the conception of the sensory experience of students within the scene. Despite that traditional media can usually be displayed only through vision and hearing, VR panoramic technology can create a multi-channel all-round virtual world, which simultaneously comprises vision, hearing, touch and smell and other multi-sensory participation in the scene, so that the students can go through a more realistic sensory world. An instance is that, in some operation steps, students need to feel the specific smells and humidity of the environment, which can trigger the relevant external devices during the interaction to spray specific gas and water vapor, can strengthen the taste and tactile sensation (The experience can only be done in the laboratory).

3) Full-scene interaction. Traditional media, generally speaking, deliver interaction with the help of such devices as screen clicks, mouses, keyboards, etc. These devices can divide the physical spaces of the location of the students and the virtual space of the location of media. The learning situation composed by VR panoramic technology allows students to wear helmets or glasses to transmit relevant interactive information in virtue of such body movements, as turning, turning eyes, gazing, etc.; and the system makes reaction to them. This enables the space of the student and the medium to be located in the same dimension. The commitment manages to consolidate the seamlessness of the experience by stimulating students' interest in learning.

\subsection{Software and Hardware Support of VR Panoramic Technology Applied to Teaching}

1) Hardware part: As a result of the updating and iteration of the hardware is updated and iterated, the price of VR equipment has become more affordable than before. The common folks, although the top VR equipment is still beyond their financial reach, have been occupying a rational space with acceptability of universities. The current price of the more professional hardware equipment is about 10,000 yuan, which can suit the demands of the majority of school students learning. Also, it also provides a greater possibility for the large-scale popularization of VR teaching environment.

2) Software part: The continuous development of VR hardware has been on the path of improving the related software. Besides, the application of software related to VR panorama has been oriented to be dominantly humanized. Moreover, with the development of AI technology, the software will be certainly featured by greater simplification and intelligence. What teachers only need to do is to pay more attention to the design of contents and interactions, not the soft- 
ware operation level.

\section{Research Outlook}

To sum up, the application of VR panoramic technology to the practical training teaching in colleges and universities is featured by overall feasibility and worthy of being explored and studied.

\subsection{It Can Reproduce the Complete Teaching Process of Practical Training}

In terms of advantages of VR panoramic technology, its realistic characteristic can portray a perfect reproduction image of the training base and the corresponding process. It is through reasonable interaction design that each step of operation is under complete simulation of the real operation process, which helps make the students' learning immersion and learning come true and go well. With the help of VR panoramic technology to teach practical training courses, students can make detailed reflection on the process and context of operation and attain prompt familiarization with the scene and operation steps by the simultaneously mastering the standard operation method, which aims to create continuous improvement on operation skills and practical competence.

\subsection{Upgrade the Teaching Effects of Practical Training Teaching}

In the actual practical training teaching process, insufficient teaching resources and equipment are known as a common phenomenon, and many students must share some resources, which accordingly limits and even reduces students' participation. VR panoramic technology provides the opportunity to every student for operating and practicing by restoring the relevant equipment and materials of the practical training base. The implementation naturally and consequentially encourages and upgrades students' participation and the sequential improvement in students' learning effect.

\subsection{Ability to Develop Personalized Teaching Experience}

Students in colleges and universities are very receptive to new things. Additionally, generally boring conceptual and principle knowledge, which can be attained through multiple channels, is not what they are concerned and interested in. instead, their interest lies in practical teaching activities. Therefore, in the practical training teaching, VR panoramic technology can invent a good practical environment and strengthen the hands-on practice process of each student by improving student participation. The implementation can fill the practical training activities with vivid and lively features, which is also in line with the psychological characteristics of college students.

The "Drilling and Completion Engineering" course has developed a practical training platform using VR panoramic technology. The results of the project have been applied to the practical and theoretical teaching of the course "Drill- 
ing and Completion Engineering", which has become an important teaching resource of the course and has been introduced into the undergraduate teaching of petroleum engineering in our university. The courses are offered on platforms such as Xuetang Online, China University MOOC and Xueyin Online, with a total number of candidates exceeding 16,000. And the effectiveness of the course teaching has been significantly improved.

On the whole, while choosing difficulty and pace, VR panoramic technology provides a digital resource, which makes learning free from longer limitation to basic memory and observation, can allow the students to operate and practice "for real" and the inaction with learning materials like a game. In addition to all kinds of practical training teaching in colleges and universities, its application scope also expands to simulating various real operation scenes which can offer students rich practical operation opportunities and resources, and realistic and immersive practical training experience. All in all, which are capable of highlighting the teaching effects of practical training, is also of great significance to study the in-depth application of VR panoramic technology in practical training teaching. Last but not the least, we should keep in mind that VR can only play its real value on the condition that the teaching method is effective. Thereby, as a college teacher, one must apply a lot of training on VR panoramic technology to the classroom and it will be the next subject we should study.

\section{Conflicts of Interest}

The authors declare no conflicts of interest regarding the publication of this paper.

\section{References}

Ge, W. R. (2018). The Design and Implementation of VR Panoramic Resources in Field Practice Classroom Teaching. Master's Thesis, Huazhong Normal University.

Ma, Z. P., Guo, B. B., Li, H. D., \& Zou, J. (2021). Pano2VR's Panoramic Roaming Implementation of Ningxia Virtual Museum. Modern Electronic Technology, 44, 149-153.

Sun, Q. (2007). The Practice and Reflection of Contextual Teaching Method in Information Technology Classroom. Network Technology Times, No. 16, 96.

Zhao, Q. (2017). Feasibility Study on the Application of VR Panoramic Technology in Information-Based Teaching. Wireless Internet Technology, No. 16, 59-60.

Zhou, Y., Yang, L. F., Zhang, Y., \& Gong, K. (2021). Construction of Experimental Teaching Platform of Medicinal Chemistry Based on VR Panoramic Technology. Guangzhou Chemical Industry, 49, 127-129. 